\title{
Drainage procedures for postoperative fluid collection after pancreaticoduodenectomy: A comparison of endoscopic ultrasound guided and percutaneous drainage
}

\author{
Da Hee WOO*, Jae Hoon LEE, Song Cheol KIM, Dae Wook HWANG, Ki Byung SONG, Jae Woo KWON, Ye Jong PARK
}

Division of Hepato-Biliary and Pancreatic Surgery, Department of Surgery, Asan Medical Center, University of Ulsan College of Medicine, Seoul, Korea

Introduction: Postoperative fluid collection (POFC) is a common complication of pancreas resection with no clear management guidelines and high morbidity rates. This study aims to analyze clinical features of patients with POFC and compare outcomes of endoscopic ultrasound (EUS) guided and percutaneous drainage in patients with POFC after pancreaticoduodenectomy (PD).

Methods: We analyzed demographic data, clinical data and intervention outcomes of 53 (EUS guided $=32$, percutaneous $=21$ ) patients who underwent PD between January 1, 2015 and June 30, 2019 at our center.

Results: Prior to drainage, $83.0 \%$ had leukocytosis and $92.5 \%$ presented with one or more of the following signs or symptoms: fever (69.8\%), abdominal pain (79.2\%), and nausea/vomiting (17.0\%). Fluid collection diameters showed a mean of $95 \mathrm{~mm}$ (range, 33 to 191 $\mathrm{mm}$ ), of which $77.4 \%$ showed a diameter decrease of more than $50 \%$ within 8 weeks of drainage $(\mathrm{EUS}=87.5 \%$ vs. PCD $=61.9 \%, p=$ 0.085). Eighty-two percent of sampled fluid collections showed positive culture results and post procedural IV antibiotics were used for an average of $8.1 \pm 4.3$ and $12.4 \pm 7.4$ days for EUS group (EG) and PCD group (PG) respectively $(p=0.012)$. EG had a shorter post-procedural hospital stay than PG $(9.8 \pm 1.1$ vs. $15.8 \pm 2.2$ days, $p=0.004)$ with no statistically significant differences in technical and clinical success rates, reintervention rates and adverse event rates.

Conclusions: EUS guided drainage (EUSD) and percutaneous catheter drainage (PCD) are both equally safe and effective in managing POFC after PD. However, EUSD was associated with shorter IV antibiotic treatment and shorter hospital stay. 